\title{
Simulación Computacional del Procedimiento de Soldadura en Servicio empleado en la Reparación Estructural de un Gasoducto
}

\author{
Héctor C. Sanzi, Gustavo F. Elvira y Alejandro M. Turel \\ Grupo de Investigación Ingeniería Estructural, Universidad Tecnológica Nacional, \\ Facultad Regional Haedo, París 532 Haedo (1706) Buenos Aires-Argentina. \\ (e-mail: tecsaing@tecsaing.com.ar)
}

Recibido Jul. 29, 2011; Aceptado Sep. 30, 2011; Versión final recibida Ago. 09, 2012

\begin{abstract}
Resumen
En este trabajo se presenta un análisis de integridad estructural del proceso de reparación de un gasoducto a partir de un modelo simplificado bidimensional usando elementos finitos. Se evalúan las tensiones residuales de origen térmico, producto del procedimiento de soldadura, considerando que se realiza con el gasoducto circulando fluido, es decir con presión aplicada. Los gasoductos que operan en Argentina son inspeccionados periódicamente de acuerdo a prácticas recomendadas por las normas del Instituto Americano del Petróleo (API) y a especificaciones, criterios y experiencias propias desarrolladas por cada planta. El modelado que se presenta permite determinar las zonas debilitadas por corrosión o por diferentes tipos de defectos o fisuras. Esto permite planificar y encausar una reparación localizada con un margen de seguridad adecuado.
\end{abstract}

Palabras clave: análisis de tensiones, elementos finitos, gasoducto, integridad estructural

\section{Computational Simulation of In-Service Welding Procedure used in the Structural Repair of a Gas Pipeline}

\begin{abstract}
In this paper, an analysis of structural integrity to the repair of a gas pipeline is presented. The study is based on a simplified two-dimensional finite elements model. The residual stresses of thermal origin caused by the welding process are evaluated considering the pipeline flowing fluid and the pressure exerted by the fluid. Pipelines in Argentina are periodically inspected following the standards of the American Petroleum Institute (API) and specifications, criteria and experiences developed by each plant. The modeling allows determining weakened areas caused by corrosion or by different types of defects or cracks. This allows planning and prosecuting a localized repair within acceptable safety margins.
\end{abstract}

Keywords: stress analysis, finite elements, gas pipeline, structural integrity 


\section{INTRODUCCION}

Las tuberías y componentes instalados en plantas de proceso, refinerías, compresoras y aquellas derivadas del petróleo, son diseñadas y construidas utilizando procedimientos adecuados y calificados mediante aporte de material por soldadura. Para ello las normas de aplicación en estructuras y recipientes contenedores de presión, American Welding Society (AWS) y American Society Mechanical Engineering (ASME IX), permiten diseñar todo tipo de uniones y calificar soldadores.

Para evitar distorsiones estructurales durante este proceso o fallas no previstas durante el servicio, los procedimientos de soldadura deberán contemplar, dependiendo del tipo de estructura, el aporte térmico incorporado, clases o tipos de materiales, medios disponibles de protección y operación, temperatura ambiente y del proceso, entre otros. Por lo tanto, es de interés para la industria, evaluar correctamente las formas de sus diseños, como son preparados y construidos.

Diferentes fenómenos metalúrgicos, cambios físicos y mecánicos se producen durante el proceso de soldadura. Es de importancia conocer y predecir dichos cambios con el objeto de asegurar la integridad de los componentes durante el servicio (Quesada et al., 2002; Jung y Tsai, 2004). Varios investigadores han desarrollado modelos analíticos y numéricos con la intención de conocer el comportamiento térmico y mecánico y especialmente, predecir las distorsiones o tensiones residuales que se desarrollan en el proceso (Nguyen et al., 1999; Anca et al.,2004; Nami et al., 2004).

Por otro lado, ensayos desarrollados en laboratorio pueden ser utilizados para ajustar dichos modelos analíticos y/o numéricos (Zalazar y Asta, 2007; Sanzi et al., 2010; Pozo Morejón et al., 2009) para su posterior utilización en el diseño. Del conjunto de uniones soldadas en gasoductos u oleoductos, aquellas que son practicadas en campo, denominadas "Soldaduras en Servicio", son las que requieren de un cuidadoso diseño y planificación, teniendo en cuenta que la tubería se encuentra con presión y fluido. En este caso, una evaluación previa, a través del uso de estos modelos analíticos y numéricos o computacionales, permitirían asegurar un diseño y metodología práctica operativa óptima, evitando distorsiones y minimizando la magnitud de tensiones residuales (Bang et al., 2002; Goldak et al.,1992).

Para estos casos, ante el planteo de un determinado procedimiento de soldadura y con el fin de conocer la magnitud de dichas tensiones o posibles distorsiones estructurales, un estudio de integridad utilizando "Técnicas Avanzadas de Análisis" a través del Método de Elementos Finitos, permitiría verificar el grado de integridad y riesgo que involucraría dicho procedimiento, simulando y analizando el proceso de reparación más eficiente, que asegure su aptitud en el servicio.

En este trabajo se presenta como primera aproximación al problema, un modelo simplificado bidimensional de Elementos Finitos, para analizar el comportamiento estructural de un gasoducto ante la reparación durante el servicio, es decir con presión y fluido, evaluando las tensiones residuales de origen térmico que se producen durante la soldadura. Modelos tridimensionales más completos y detallados (Alberg y Berglund, 2004), incluyendo cambios de las variables físicas que intervienen en el proceso ante los cambios de fase, variación de la densidad, calor específico y conductividad con la temperatura (Sanzi y Elvira, 2009), permitirían ajustar las hipótesis simplificativas propuestas en el modelo.

\section{APTITUD PARA ELSERVICIO DE LINEAS DE TRANSPORTE DE GAS Y PETROLEO}

En nuestro país, las cañerías para el transporte de gas son diseñadas mediante la norma (ANSI/ASME B31.8). En servicio estos componentes son inspeccionados periódicamente, en acuerdo a prácticas técnicas utilizadas para tal fin, como son las normas American Petroleum Institute (API), en conjunto con especificaciones, criterios y experiencias propias desarrollados por cada planta. En este caso, es imprescindible contar con una correcta planificación de la inspección ante la posibilidad de encontrar zonas debilitadas por efecto de la corrosión u otros defectos y dependiendo del grado de riesgo de la línea, de su contenido y considerando las variables 
operativas presentes, un estudio de "Inspección Basada en Riesgo" (API 581), permite minimizar el grado de riesgo en la operación.

Ante la presencia de esta anormalidad estructural, se debe planificar la reparación adecuada que consiste en el cambio del tramo defectuoso por uno nuevo o eventualmente colocar un refuerzo provisorio, es decir la colocación de una camisa alrededor de la sección dañada, "Encamisado", con un material de características similares y permitiendo de esta manera recuperar estructuralmente la pérdida de espesor o recubrir el sector dañado.

Este tipo de reparación se denomina "Soldadura en Servicio" y se utiliza tanto en reparación como en extensión de líneas, con el objeto de mantener el margen de seguridad correcto que requieren estos sistemas según se citan, entre otras, en las referencias (Manual Pipelines Repairs, 1995; Seleznev et al, 2005; Pipe Engineering Tube Turns, 1986). En esta situación y desde el punto de vista de la integridad y el riesgo, es fundamental conocer el nivel tensiones residuales que se alcanza en la unión, producto del proceso de soldadura. Esta magnitud se debe adicionar a las tensiones en la tubería, producto de los distintos estados de carga.

El procedimiento constructivo, en total acuerdo con el Apéndice B de la Norma (API 1104), facilita la calificación del procedimiento necesario para asegurar una ejecución adecuada.

Dicho proceso de calificación, consiste en hacer circular agua sobre una tubería, mientras se realiza la reparación o encamisado, midiendo con termocuplas la distribución de temperaturas que se desarrollan durante el proceso, permitiendo de esta manera seleccionar el más adecuado y calificar a los soldadores. En la Fig.1 se presenta una fotografía de una reparación en un tramo de una cañería para el transporte de gas.

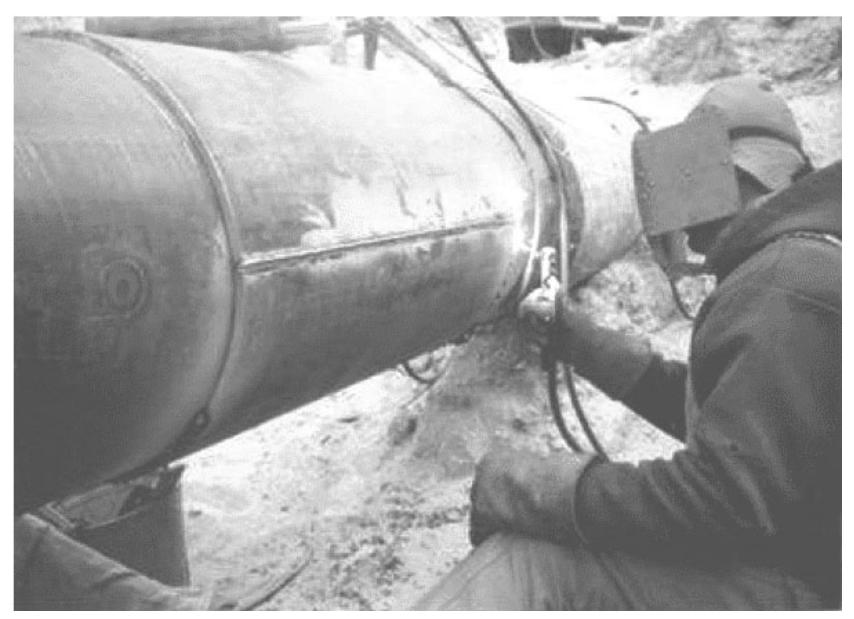

Fig.1: Reparación en un tramo de Gasoducto

Teniendo en cuenta los conceptos enunciados, y ante la necesidad de determinar la magnitud de las tensiones residuales, un estudio detallado de integridad utilizando "Técnicas Avanzadas de Análisis" a través del Método de Elementos Finitos (Bang et al., 2002; Otegui et al., 2000), permitiría verificar el grado de integridad y riesgo estructural que involucraría dicha falla 0 debilitamiento, simulando y analizando el proceso de reparación más eficiente que asegure su aptitud en el servicio, hasta tanto no se decida el recambio

Por lo tanto, previo al estudio de integridad y con el objeto de validar modelos de cálculos futuros, aplicables para cada caso y que sean utilizados como patrones de referencia, se plantea un modelo simplificado bidimensional de Elementos Finitos para simular el procedimiento de calificación de la reparación por soldadura, haciendo circular agua a temperatura ambiente por el interior de la cañería. Los resultados de la simulación se podrían comparar con las temperaturas medidas mediante termocuplas (Zalazar y Asta, 2007). De esta manera se posibilitaría el ajuste del modelo propuesto. 


\section{GEOMETRIA DE DETALLE DE LOS ELEMENTOS ESTRUCTURALES QUE CONFORMAN LA UNION}

Dimensional y material de la cañería

La norma de diseño (ANSI/ASME B31.8) y que cubre la construcción, es utilizada para la determinación del espesor de gasoductos.

El gasoducto en estudio presenta un diámetro nominal de DN16", espesor 11.5 milímetros y material acero al carbono calidad tipo API 5L Grado X60, sin costura, con composición química,

Tabla 1: Composición Química de API 5L Gr X60

\begin{tabular}{|c|c|c|c|c|c|}
\hline & Cmax \% & Mn max \% & $\mathrm{P} \max \%$ & $\mathrm{~S} \max \%$ & Si max \% \\
\hline API 5L Gr X60 & 0.13 & 1.2 & 0.007 & 0.001 & 0.27 \\
\hline
\end{tabular}

Procedimiento de la reparación

El material del refuerzo tipo "Encamisado", de ancho $400 \mathrm{~mm}$, es del tipo ASTM A516 Gr70. El procedimiento de reparación se basa en los lineamientos dados en la norma (API 1104). En la Fig. 2, se presenta un esquema constructivo y dimensional de la zona de reparación.

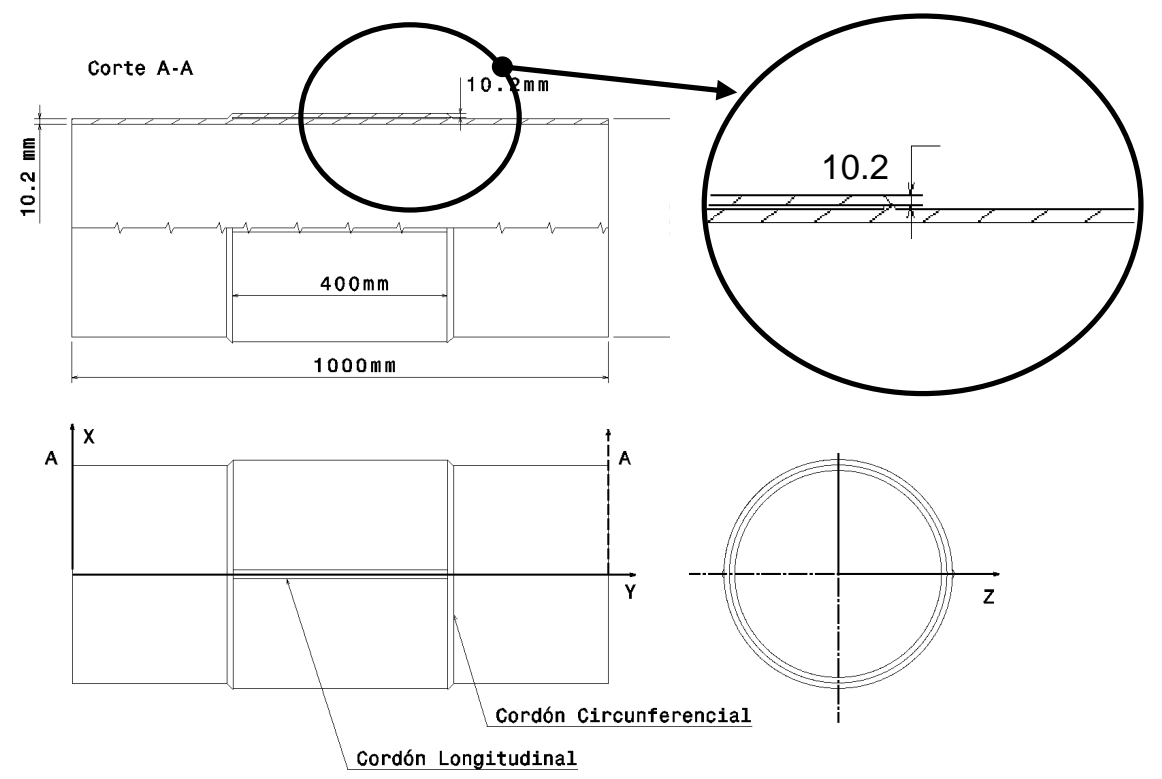

Fig. 2: Esquema dimensional de la reparación

El procedimiento de soldadura que se aplica al cordón circunferencial es del tipo manual "SMAW", y consiste en la realización de cuatro pasadas de material de aporte, utilizando un electrodo ASTM E7018. El calor aportado (Q), depositado en la unión de las dos medias cañas del "Encamisado", esta relacionado directamente con la tensión y corriente de la máquina, la cual debe encontrarse en perfecto estado, utilizando una eficiencia o rendimiento del proceso, de valor 0.85. El aporte térmico, en términos de potencia disipada efectiva, se obtiene utilizando las expresiones dadas en la Bibliografía (Fundamentals of Heat Transfer, 1980).

La secuencia propuesta para el procedimiento de soldadura, consiste en la realización de cuatro capas o pasadas, es decir la ejecución completa de cuatro cordones de soldadura. El estudio esta centralizado en el análisis del comportamiento térmico y estructural sobre los cordones de soldaduras circunferenciales, ya que los longitudinales entre ambas mitades de la camisa, no presentan un riesgo estructural. 


\section{VALIDACION DEL MODELO COMPUTACIONAL}

Previo al estudio de integridad estructural y con el objeto de validar el modelo de cálculo empleado, se simula el procedimiento de calificación de la reparación por soldadura, en acuerdo al Apéndice B de las Normas (API 1104), donde se hace circular agua a temperatura ambiente por el interior de la cañería. Los resultados obtenidos se comparan con los publicados en un trabajo previo de referencia (Sanzi et al., 2010).

Simulación geométrica de los elementos que conforman la unión, incluyendo el cordón de soldadura

Teniendo en cuenta la simetría de revolución que presentan los elementos que conforman la zona de reparación (gasoducto y refuerzo), respecto de un plano transversal, se propone un modelo localizado y altamente densificado, utilizando elementos del tipo axil-simétricos isoparamétricos de 8 nodos PLANE2D, con el objeto de obtener resultados más precisos. El modelo quedó conformado por 7775 nodos y 6963 elementos, Fig. 3.

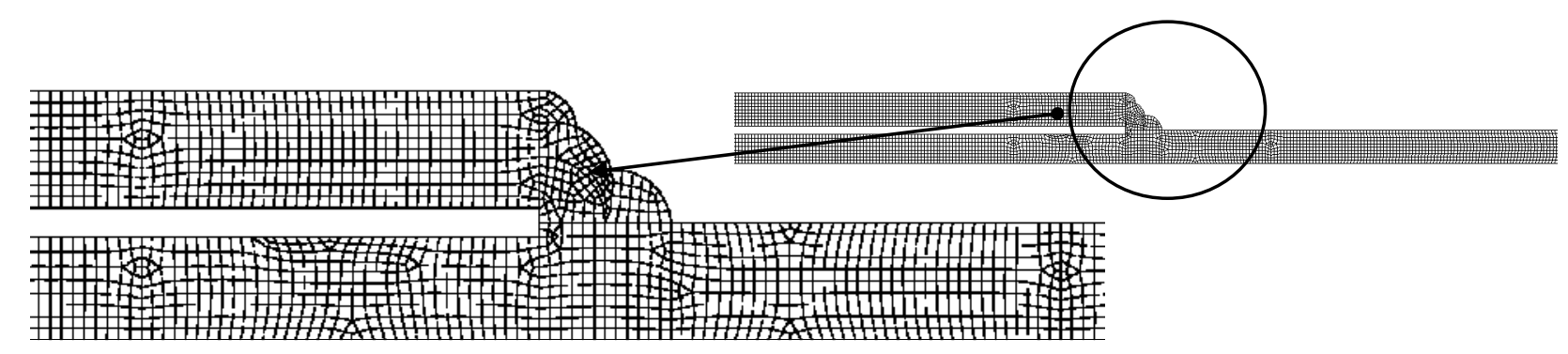

Fig. 3: Modelo de Elementos Finitos del Gasoducto

Para el estudio de integridad del gasoducto, se debería considerar la situación estructural más desfavorable, que consiste en utilizar los espesores corroídos del tramo dañado o debilitado, y justamente en aquellas zonas del componente que se encuentra en contacto con el gas, incluyendo el interior del refuerzo, suponiendo que el defecto es pasante.

\section{Simulación del proceso de soldadura}

El proceso de soldadura es representado a través de la energía aportada por cada cordón completo, en su recorrido circunferencial. La simulación del proceso de soldadura consiste en:

1.- Para la primer pasada, el cordón es modelado por 47 elementos (volumen que representa la cantidad de material de aporte y la potencia calórica que ingresa al modelo), identificados con un punto en la Fig. 4. Así sucesivamente se repite el concepto para el resto de los cordones.

2.- El proceso de avance de cada cordón completo se representa mediante funciones temporales, adecuadamente definidas y que actúan en forma sucesiva en los Volúmenes de Carga, activando y desactivando estos volúmenes de acuerdo a la secuencia programada, los cuales en su conjunto, conforman o representan el aporte de soldadura.
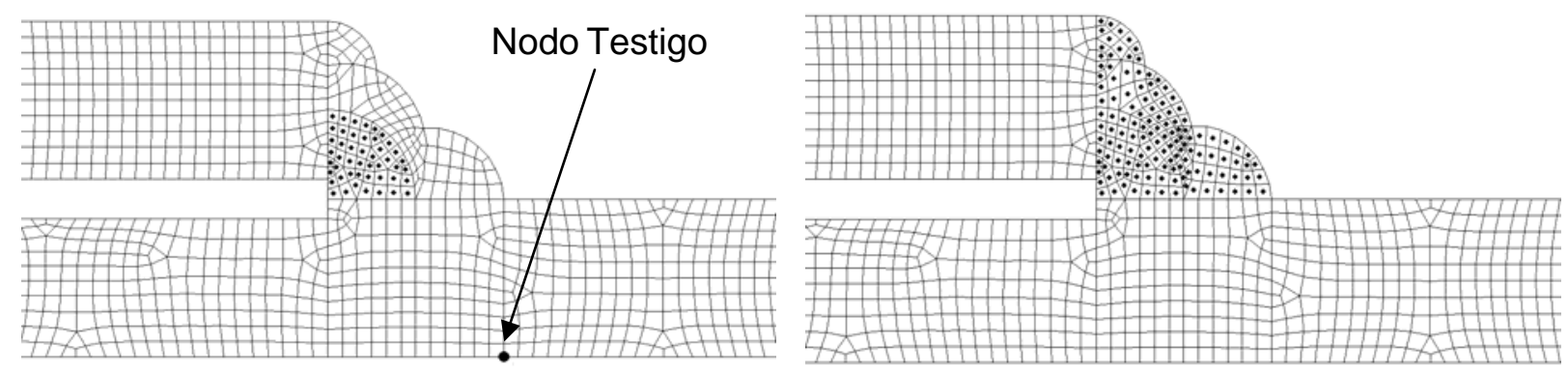

Fig. 4: Generación interna de calor en zona de aporte $-1^{\mathrm{er}}$ y $4^{\text {to }}$ cordón. Punto "Test" 
Como primera aproximación se considera que el mecanismo de transferencia de calor esta dado por efectos de conducción y convección, despreciando los fenómenos de radiación. La conductividad y calor específico se consideran constantes con la temperatura.

A partir de la simulación, los resultados obtenidos de la distribución de temperaturas, para un nodo identificado como "Punto Test" coincidentes con la ubicación de la termocupla, según publicación en referencia (Sanzi et al., 2010), son del mismo orden, lo cual demuestra que el modelo planteado es apto para su utilización en la evaluación del nivel de tensiones.

\section{ANALISIS DE INTEGRIDAD ESTRUCTURAL “STRESS ANALYSIS” DEL GASODUCTO}

Con el modelo validado, se analiza la integridad estructural en la zona reparada del gasoducto, teniendo en cuenta todos los estados de carga presentes, es decir la carga térmica, producto del procedimiento de soldadura y la presión interior, en la condición corroída.

Para simular la reparación, se consideran las condiciones reales que se presentan durante dicho proceso, es decir para ambos lados de la cañería, del tipo convectivas, circulando gas en el interior y aire calmo en el exterior. Se plantea que en el instante inicial de tiempo, la temperatura es ambiente.

De la historia térmica, se debe elegir aquella distribución que mayor gradiente produzca sobre la unión, es decir la distribución que ocasione mayores distorsiones cuando el gasoducto se enfríe. En ese instante y para una distribución dada, se calculan las tensiones térmicas, que se superponen con las obtenidas por presión interior. En la Fig. 5 se presenta la distribución de temperaturas que serán consideradas para la evaluación de tensiones térmicas.
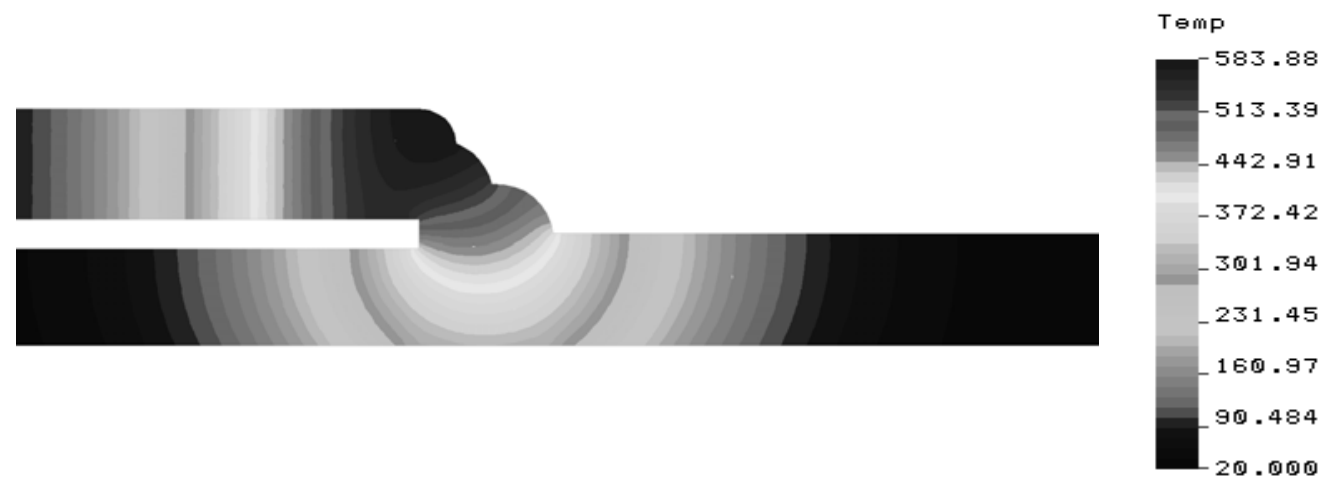

Fig. 5: Distribución de temperatura a considerar en la evaluación de tensiones térmicas

En la Fig. 6 se presentan las tensiones de Von Mises, producto de la combinación de ambos estados de carga.

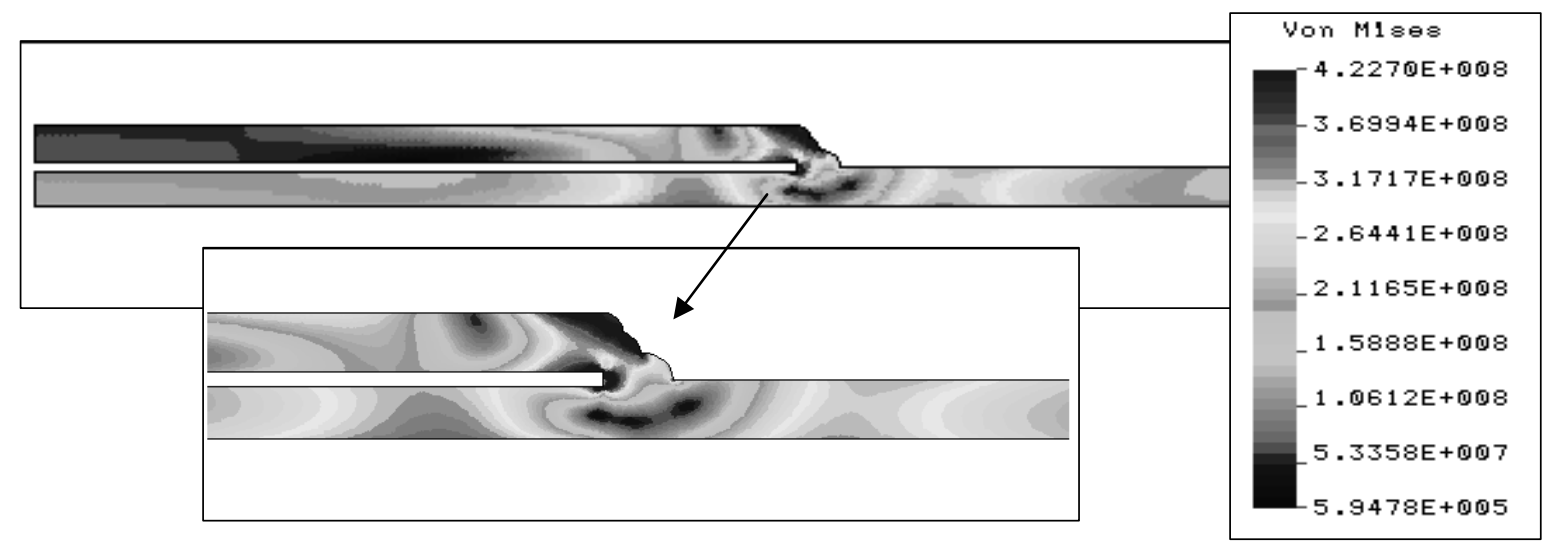

Fig. 6: Estado de tensiones de Von Mises, para gradiente térmico máximo ( $\mathrm{Pa})$ 


\section{DISCUSION DE RESULTADOS}

Se aprecia una gran coincidencia de la distribución de temperaturas o isotermas, obtenidas a partir del modelo propuesto, con los que resultaron de las mediciones con termocuplas, (Sanzi, et al., 2010).

Se evaluaron las Tensiones Primarias de Membrana y Primarias más Secundarias, en los elementos que conforman la zona de reparación, aplicando el "Criterio de Clasificación de Tensiones" del Código (ASME Sección VIII División 2), considerando el estado de carga térmico, del cual se desprende, a partir de los resultados presentados en la Figura 6, que las tensiones de Von Mises se mantienen con valores inferiores a los admisibles, lo cual asegura la integridad del componente.

\section{CONCLUSIONES}

En este trabajo se ha presentado un estudio de Integridad y Riesgo Estructural, a través de un sencillo modelo Bidimensional de Elementos Finitos (Sólido de Revolución), que simula un procedimiento de reparación de un sector dañado de un gasoducto mediante una "Soldadura en Servicio", y donde se incluyeron los efectos de la presión interior.

Previamente, el modelo fue validado utilizando los resultados obtenidos en un trabajo anterior, publicado en referencia (Sanzi et al., 2010), donde se comparó la distribución térmica final con los de un ensayo de soldadura del laboratorio, con medición de termocuplas.

Es importante entonces, desarrollar soluciones a problemas de integridad en reparaciones de gasoductos, aplicando un procedimiento de análisis sistemático y seguro, previos a ensayos de laboratorio, que permitan a especialistas en soldadura e inspectores, aplicar y optimizar procedimientos, reduciendo riesgos y costos.

Un estudio como el presentado, utilizando "Técnicas Avanzadas de Análisis", permite verificar el grado de integridad y riesgo que involucra una reparación en campo, simulando y analizando el proceso más eficiente, que asegure su aptitud durante el servicio.

Este procedimiento de cálculo ha proporcionado resultados con un grado aceptable de exactitud pero con el objeto de ajustar el grado de detalle en dichos resultados, actualmente se han desarrollado modelos tridimensionales más complejos, e incorporando en su formulación, la variación de la conductividad y el calor específico con la temperatura.

\section{REFERENCIAS}

Alberg H. y Berglund D., Comparison of an axisymmetric and a three-dimensional model for welding and stress relief heat treatment. 8th International Conference on Numerical Methods in Industrial Forming Processes, NUMIFORM. Columbus, Ohio, USA. June (2004).

Anca A., Cardona A., y Risso J.M. 3D-Thermo-mechanical simulation of welding processes. Mecánica Computacional Vol. XXIII. 2301-2318, (2004).

ANSI/ASME B31.8, Gas Transmission and Distribution Piping Systems, 212. New York. USA (2010).

API 1104. Welding of Pipelines and Related Facilities, Pipeline Segment, 123. Washington, D.C. USA (2010).

API RP 581. Risk-Based Inspection Technology. Ed. 2nd, 607. Washington, D.C. USA (2008).

ASME Boiler \& Pressure Vessel Code - Section VIII - Pressure Vessels (VIII-DIV 1, VIII-DIV 2). New York. USA (2010). 
ASME Boiler and Pressure Vessel Code - Section IX: Welding and Brazing Qualifications, 350. New York. USA (2010).

Bang I.W., Son Y.P., Oh K.H., Kim Y.P. y .Kim W.S, Numerical simulation of sleeve repair welding of inservice gas pipelines, Welding Research Journal, 273-282. (2002).

Goldak, J A., Oddy, A S., Dorling, D V, Finite element analysis of welding on fluid filled, pressurized pipelines. 45-50. International Trends in Welding Science and Technology. Gatlinburg, Tennessee. USA. June (1992).

Jung G. y Tsai C., Plasticity-based distortion analysis for fillet welded thin-plate t-joints. Welding Journal 83 (6), 177-187. (2004).

Lindon Thomas, Fundamentals of Heat Transfer, $1^{\text {st }}$ ed, 720. Prentice-Hall Inc., Englewood Cliffs, NJ, USA. (1980).

Manual Pipelines Repairs (Supplements to ANSI/ASME B31.4 and B31.8 ). SHELL Int. 36. (1995).

Nami M., Kadivar M., y Jafarpur K., Three-dimensional thermal response of thick plate weldments: effect of layer-wise and piece-wise welding. Modelling Simul. Mater. Sci. Eng. 12. 731-743 (2004).

Nguyen, Ohta, Matsuoka, Suzuki y Taeda, Analytical solutions for transient temperature of semi-infinite body subjected to 3D moving heat sources, Welding Research 78 (August), 265-273. (1999).

Otegui J.L., Urquiza S., Rivas, Trunzo A, Local colapse of gas pipelines under sleeve repairs, Pressure vessel and piping. ED. ELSEVIER 7 (9), 555-566 (2000).

Pipe Engineering. $6^{\text {th }}$ ed, 224. Tube Turns, Inc. Louisville, Kentucky, USA. (1986).

Pozo Morejón J. A., García Jacomino J. L., Ramos Morales F., García Rodríguez Y., Cruz Crespo A., Díaz Cedré E. M., Duffus Scott A. B., Methodology of modelation with ANSYS of the thermal history, stress and strain caused by welding. Revista de la Facultad de Ingeniería, Universidad Central de Venezuela. 24 (2). 13-26. (2009).

Quesada H.J., Salazar M, Asta E.P., Ensayo de fisuración en frío, aplicados en aceros de alta resistencia. Decimocuartas Jornadas Técnicas de Soldadura, 333-340. Madrid. España. Junio (2002).

Sanzi H. y Elvira G., Evaluación del ciclo térmico de un proceso de soldadura tipo GMAW, en chapas de acero, mediante el método de elementos finitos. Análisis de la influencia de las variables físicas del acero, Congreso SAM/CONAMET, Anales del Congreso. Bs As, Argentina. Octubre (2009).

Sanzi H., Elvira G., y Turel A., Evaluación del procedimiento de soldadura mediante simulación computacional en la reparación estructural de un gasoducto. $5^{a}$ Conferencia sobre Usos del Acero, Jornada de Análisis de Falla y Prevención 2010 y Jornada de Soldadura, IAS. Anales del Congreso. Rosario. Argentina. (2010).

Seleznev V., Aleshin V. y Kobyakov V., Analysis of the corroded pipeline segments using in line inspection data. The $8^{\text {th }}$ International Conference of Slovenian Society for Non-Destructive Testing, Aplication of contemporary Non-Destructive testing in Engineering. 383-389. Portoroz, Slovenia. September (2005).

Salazar M. y Asta E., Análisis de soldaduras en servicio para cañerías aplicando diferentes procesos de arco. ESAB_CONARCO Año XXX (128), 14-19. (2007). 